\title{
Sleep timing and vegetable intakes in UK adults: a cross-sectional study
}

\section{Lack of sleep is increasingly a topic of public health concern, and evidence suggests it could be a modifiable obesity risk factor ${ }^{(1)}$. Fruit and vegetable $(\mathrm{FV})$ intakes also influence bodyweight and chronic disease risk ${ }^{(2)}$. Few studies have investigated the associations between sleep measures and FV intakes. We therefore assessed the associations between sleep, FV intakes and biomarkers of FV intakes. \\ Data from 240 healthy adults collected to validate an online dietary assessment tool (myfood24) ${ }^{(3)}$ was used for these analyses.} Sleep (onset, offset, duration and mid-sleep time) were measured by SenseWear ${ }^{\mathbb{B}}$ armbands. FV groups (servings/day) based on the National Diet and Nutrition Survey categories ${ }^{(4)}$ were assessed using the Oxford WebQ online dietary questionnaire ${ }^{(5)}$. Plasma concentrations of total vitamin $\mathrm{C}$ and $\beta$-carotene were used as biomarkers of FV intakes. Regression analyses were used to determine the associations between sleep variables (exposures) with FV intakes and their associated biomarkers (outcomes), adjusting for age, sex, socio-economic status, ethnicity, smoking and total energy intake. All outcome variables were log-transformed. Restricted cubic splines (RCS) were also used to model the associations.

Sleep duration was not associated with FV intakes and their associated biomarkers. However, sleep timing was associated with FV intakes. Every hour later sleep offset (waking time) was associated with $10 \%$ (exp (b) $=0.90,95 \%$ CI 0.82 to $0.99, p=0.03$ ) lower intakes of fruit. Similarly, every hour later mid-sleep time was associated with $9 \%(\exp (b)=0.91,95 \%$ CI 0.83 to $0.99, p=0.04)$ lower intakes of total fruit, and RCS modelling showed that the association was linear $(p=0.09)$ (Figure 1). Although, there were no associations between sleep duration and total vegetable intakes, every hour later sleep onset was associated with $8 \%$ (exp (b) $=1.08,95 \%$ CI 1.01 to $1.16, p=0.01$ ) higher intakes of other vegetables (e.g. beetroot, cucumber, lettuce, peas, celery and leeks) and every hour later sleep offset was associated with $9 \%(\exp (b)=1.09,95 \% \mathrm{CI} 1.002$ to $1.18, p=0.04)$ higher intakes of other vegetables. RCS modelling showed that the positive association between sleep onset and other vegetables was linear $(\mathrm{p}=0 \cdot 1)$ (Figure 2). Every hour later mid-sleep time was associated with $11 \%(\exp (b)=1.11,95 \%$ CI 1.02 to $1.21, p=0.01)$ higher intakes of other vegetables. In conclusion, these results show that every hour later of sleep onset, offset and mid-sleep time are associated with higher intakes of other vegetables. Larger studies are required to confirm these findings.

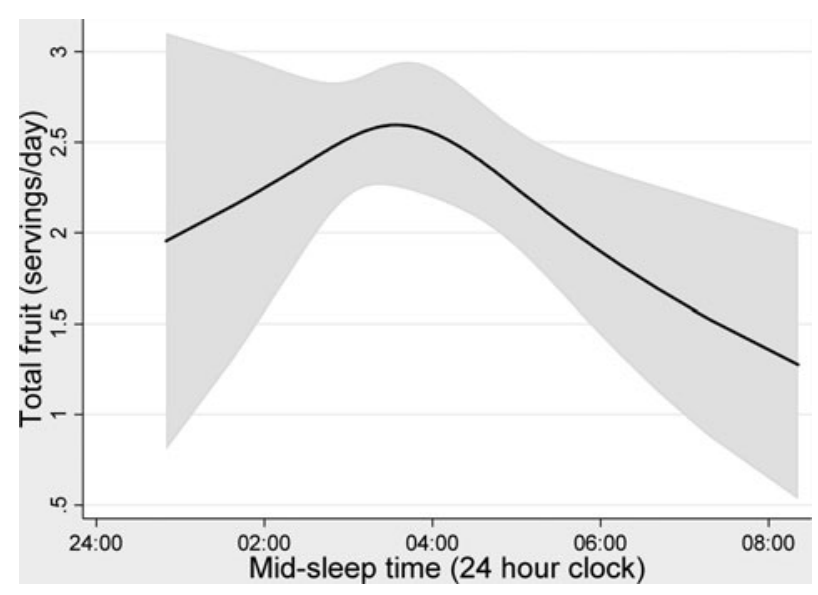

Fig. 1. Mid-sleep time and total fruit

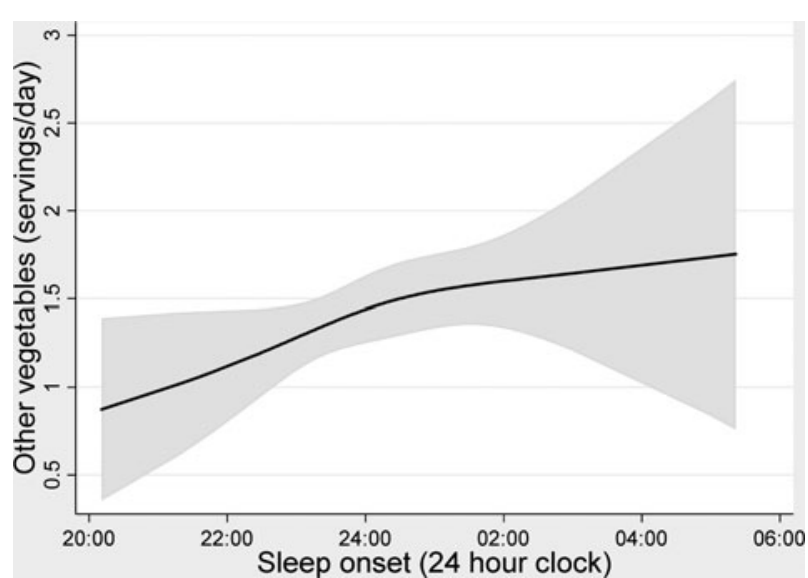

Fig. 2. Sleep onset and other vegetables

E.A.N is in receipt of a scholarship from Umm Al-Qura University, Saudi Arabia. G.D.M.P is supported by a Medical Research Council Doctoral Training Grant.

1. Wu Y, Zhai L, \& Zhang D (2014) Sleep Med 15, 1456-1462.

2. Aune D, Giovannucci E, Boffetta P et al. (2017) Int J Epidemiol 46, 1029-1056.

3. Carter MC, Albar SA, Morris MA et al. (2015) Nutrients 7, 4016-4032.

4. Fitt E, Mak TN, Stephen AM et al. (2010) Eur J Clin Nutr 64 Suppl, S32-S36.

5. Liu B, Young H, Crowe F et al. (2011) Public Health Nutr 14, 1998-2005. 